\title{
Determining skeletal maturation stage using cervical vertebrae: evaluation of three diagnostic methods
}

Luci Mara Fachardo Jaqueira ${ }^{(a)}$ Mônica Costa Armond(a)

Luciano José Pereira ${ }^{(a)}$ Carlos Eduardo Pinto de Alcântara ${ }^{(b)}$

Leandro Silva Marques(a)

(a) Department of Orthodontics, School of Dentistry, Vale do Rio Verde University, Três Corações, MG, Brazil.

(b) School of Dentistry, Federal University of Vales do Jequitinhonha e Mucuri, Diamantina, MG, Brazil.
Corresponding author:

Leandro Silva Marques

Rua Arraial dos Forros, 215

Diamantina - MG - Brazil

CEP: 39100-000

E-mail: Ismarques21@yahoo.com.br

Received for publication on Jun 07, 2010 Accepted for publication on Jul 07, 2010

\begin{abstract}
The aim of the present study was to compare the use of three cervical vertebral evaluation methods (Hassel-Farman, Baccetti et al., and Seedat-Forsberg) for determinating skeletal maturation stage in orthodontic patients. Twenty-three radiographs were randomly selected from a private orthodontic practice. Each radiograph was analyzed on three separate occasions by four evaluators (one radiologist and three orthodontists), who determined the skeletal maturation stage using the references established by each of the three methods. Intraevaluator and interevaluator comparisons were performed, and the degree of agreement was established using the weighted Kappa coefficient (95\% CI). Good agreement (Kappa between 0.61 and 0.80 ) was observed between the determinations of most of the evaluators. The three methods demonstrated clinical applicability. However, the method proposed by Baccetti et al. achieved the best results, followed by the Hassel-Farman and the SeedatForsberg methods.
\end{abstract}

Descriptors: Growth and Development; Cervical Vertebrae; Orthodontics.

\section{Introduction}

The most frequently employed methods for determining skeletal maturation stage in orthodontics are the use of radiographs of the hand and wrist, also the cervical vertebrae. A number of authors state that such methods are reliable and that they can be routinely employed in orthodontic treatment planning. ${ }^{1-7}$

Recent studies have shown that patients might benefit more by assessing $\mathrm{x}$-rays of the cervical vertebrae than the hand and wrist. ${ }^{6,7}$ The main argument is that evaluation of the vertebrae can be made from routine orthodontic documentation, thereby avoiding the second dose of radiation necessary for radiography of the hand and wrist.

The chief methods for determining skeletal maturation in orthodontic patients through the assessment of cervical vertebrae are those proposed by Hassel and Farman ${ }^{8}$ (1995), Baccetti et al. ${ }^{9}$ (2002), and Seedat and Forsberg $^{10}$ (2005). Although these methods have been systematically employed in studies and clinical practice, the literature does not offer any consistent evidence regarding their advantages and disadvantages or the limitations of one method compared with another.

Thus, the aim of this study was to compare three cervical vertebral 
evaluation methods (Hassel-Farman, ${ }^{8}$ Baccetti et al., ${ }^{9}$ and Seedat-Forsberg ${ }^{10}$ ) for determining skeletal maturation stage in orthodontic patients.

\section{Materials and Methods}

Twenty-three radiographs of good quality and no overlapping of the cervical vertebrae were randomly selected. These were diagnostic radiographs of patients never having had any type of orthodontic or orthopedic treatment, had no congenital or acquired malformation of the cervical vertebrae, nor having any developmental abnormalities. The patients were between 7 and 49 years of age and therefore in different stages in relation to the adolescent growth spurt.

The study was based on identifying the skeletal maturation stage of the cervical vertebrae by four evaluators (three orthodontists and one radiologist) who were duly instructed in performing the evaluation using the three proposed methods. Each examiner received a folder containing the 23 radiographs with patient data blacked out as well as a pencil sharpener, eraser, ruler, and an explanatory text with drawings illustrating the three cervical vertebral maturation identification methods. Each evaluator was instructed to first perform all evaluations using the Hassel-Farman method, ${ }^{8}$ followed by the Baccetti et al. method, ${ }^{9}$ and finally the Seedat-Forsberg method. ${ }^{10}$ There was a 15 -day interval between evaluations with the aim of eliminating possible influences of one method over the other. Radiograph inspection was performed using an $\mathrm{x}$-ray film viewer with standard light intensity and the use of a mask to isolate the radiograph and limit excess light.

In the Hassel-Farman method, ${ }^{8}$ there are six cervical vertebral maturation stages (CVMSs): initiation, acceleration, transition, deceleration, maturation, and finalization. The method proposed by Baccetti et al. ${ }^{9}$ has five CVMSs, and it allows two variations for each stage. In the method proposed by Seedat and Forsberg, ${ }^{10}$ the authors use the six stages proposed by Hassel and Farman, ${ }^{8}$ but evaluate changes occurring only in the body of the third cervical vertebra (C3). The authors justify the option for the $\mathrm{C} 3$ vertebra for its easier visualization in the cephalogram. The S-shape on the upper edge of C3 is indicative of active adolescent growth, occurring in the initiation and acceleration stages. The lipshape on the lower edge of C3 occurs in the transition and deceleration stages, whereas the rounding of the lower corners of the vertebra suggests little growth or the end of growth, corresponding to the maturation and finalization stages.

For comparison purposes, an adjustment was made to allow for the Hassel-Farman method having six CVMSs and the method proposed by Baccetti et al. ${ }^{9}$ having five (two variants for each stage, with the exception of Stage III). Thus, all patients determined to be in Stage VI of the Hassel-Farman ${ }^{8}$ and Seedat-Forsberg ${ }^{10}$ methods were considered to be in Stage V. We deemed this adjustment to be valid, as there is a form of Hassel-Farman ${ }^{8}$ Stage VI in Stage V of the Baccetti et al. method. ${ }^{9}$

There is no "gold standard" for estimating skeletal maturation through the use of cervical vertebrae. Therefore, a radiologist was used in this study as a

Table 1 - Interexaminer agreement (radiologist vs. orthodontists) for the Hassel-Farman method, ${ }^{8}$ expressed as skeletal maturation stage.

\begin{tabular}{|c|c|c|c|c|c|c|}
\hline \multicolumn{6}{|c|}{ Radiologist } & \multirow{2}{*}{ Weighted Kappa (95\% Cl) } \\
\hline Stages & 1 & 2 & 3 & 4 & 5 & \\
\hline \multicolumn{6}{|c|}{ Orthodontist 1} & \multirow{6}{*}{$0.64(0.45-0.83)$} \\
\hline 1 & 3 & 0 & 0 & 0 & 0 & \\
\hline 2 & 0 & 3 & 1 & 0 & 0 & \\
\hline 3 & 0 & 2 & 3 & 0 & 0 & \\
\hline 4 & 0 & 0 & 2 & 0 & 1 & \\
\hline 5 & 0 & 0 & 2 & 3 & 3 & \\
\hline \multicolumn{6}{|c|}{ Orthodontist 2} & \multirow{6}{*}{$0.69(0.50-0.88)$} \\
\hline 1 & 3 & 1 & 0 & 0 & 0 & \\
\hline 2 & 0 & 4 & 1 & 0 & 0 & \\
\hline 3 & 0 & 0 & 4 & 0 & 1 & \\
\hline 4 & 0 & 0 & 2 & 3 & 2 & \\
\hline 5 & 0 & 0 & 1 & 0 & 1 & \\
\hline \multicolumn{6}{|c|}{ Orthodontist 3} & \multirow{6}{*}{$0.76(0.58-0.93)$} \\
\hline 1 & 3 & 0 & 1 & 0 & 0 & \\
\hline 2 & 0 & 3 & 2 & 0 & 0 & \\
\hline 3 & 0 & 2 & 4 & 0 & 0 & \\
\hline 4 & 0 & 0 & 1 & 3 & 1 & \\
\hline 5 & 0 & 0 & 0 & 0 & 3 & \\
\hline
\end{tabular}


reference point, being the most experienced among the evaluators as well as a researcher in the field of imaging diagnostics. After we collected the data, the weighted Kappa test was performed to assess the degree of intrareference examiner (radiologist) agreement and interexaminer agreement between the radiologist and the three orthodontists. Orthodontist 1 had 4 years of clinical experience, whereas orthodontists 2 and 3 had 9 and 15 years of experience, respectively.

The data were analyzed using the Stata computer program (Stata, Version 9.0, Stata Corp., College Station, TX, USA) and the degree of agreement was obtained based on the Altman classification: $^{11} \quad 0.00-0.20=$ very low; $0.21-0.40=$ low; $0.41-0.60=$ fair; $0.61-0.80=$ good $;$ and $0.81-1.00=$ very good. The confidence interval $(\mathrm{CI})$ was $95 \%$. The study was approved by the Ethics Committee of the University of Vale do Rio Verde, Faculty of Dentistry.

Table 2 - Interexaminer agreement (radiologist vs. orthodontists) for the Baccetti et al. method ${ }^{9}$ expressed as skeletal maturation stage.

\begin{tabular}{|c|c|c|c|c|c|c|}
\hline \multicolumn{6}{|c|}{ Radiologist } & \multirow{2}{*}{ Weighted Kappa (95\% CI) } \\
\hline Stages & 1 & 2 & 3 & 4 & 5 & \\
\hline \multicolumn{6}{|c|}{ Orthodontist 1} & \multirow{6}{*}{$0.75(0.57-0.94)$} \\
\hline 1 & 3 & 0 & 0 & 0 & 0 & \\
\hline 2 & 1 & 5 & 0 & 0 & 0 & \\
\hline 3 & 0 & 1 & 3 & 0 & 0 & \\
\hline 4 & 0 & 1 & 1 & 5 & 0 & \\
\hline 5 & 0 & 0 & 1 & 1 & 1 & \\
\hline \multicolumn{6}{|c|}{ Orthodontist 2} & \multirow{6}{*}{$0.73(0.56-0.90)$} \\
\hline 1 & 3 & 1 & 0 & 0 & 0 & \\
\hline 2 & 1 & 5 & 1 & 0 & 0 & \\
\hline 3 & 0 & 1 & 2 & 2 & 0 & \\
\hline 4 & 0 & 0 & 2 & 4 & 0 & \\
\hline 5 & 0 & 0 & 0 & 0 & 1 & \\
\hline \multicolumn{6}{|c|}{ Orthodontist 3} & \multirow{6}{*}{$0.73(0.56-0.90)$} \\
\hline 1 & 3 & 1 & 0 & 0 & 0 & \\
\hline 2 & 1 & 6 & 2 & 0 & 0 & \\
\hline 3 & 0 & 0 & 1 & 2 & 0 & \\
\hline 4 & 0 & 0 & 2 & 4 & 0 & \\
\hline 5 & 0 & 0 & 0 & 0 & 1 & \\
\hline
\end{tabular}

\section{Results}

Agreement comparing the three methods with one another, as determined by the principal examiner (radiologist) was considered good (>0.64). Table 1 shows good agreement between the principal examiner (radiologist) and the three orthodontist examiners $(0.64,0.69$, and 0.76 , respectively) for the Hassel-Farman method. ${ }^{8}$

There was also good agreement between the radiologist and the three orthodontist examiners concerning the Bacceti et al. method $^{9}(0.75,0.73$, and 0.73 , respectively) (Table 2) and the Seedat-Forsberg $\operatorname{method}^{10}(0.63,0.66$, and 0.54 , respectively) (Table 3).

\section{Discussion}

Results of our study indicate that determination of skeletal maturation stage via cervical vertebrae had satisfactory clinical applicability, within the limits of this study, using any of the three principal

Table 3 - Interexaminer agreement (radiologist vs. orthodontists) for the Seedat-Forsberg method ${ }^{10}$ expressed as skeletal maturation stage.

\begin{tabular}{|c|c|c|c|c|c|c|}
\hline \multicolumn{6}{|c|}{ Radiologist } & \multirow{2}{*}{ Weighted Kappa (95\% Cl) } \\
\hline Stages & 1 & 2 & 3 & 4 & 5 & \\
\hline \multicolumn{6}{|c|}{ Orthodontist 1} & \multirow{6}{*}{$0.63(0.43-0.82)$} \\
\hline 1 & 3 & 0 & 0 & 0 & 0 & \\
\hline 2 & 2 & 3 & 0 & 0 & 0 & \\
\hline 3 & 0 & 4 & 3 & 1 & 0 & \\
\hline 4 & 0 & 0 & 0 & 1 & 0 & \\
\hline 5 & 0 & 0 & 2 & 1 & 3 & \\
\hline \multicolumn{6}{|c|}{ Orthodontist 2} & \multirow{6}{*}{$0.66(0.43-0.89)$} \\
\hline 1 & 4 & 0 & 0 & 0 & 0 & \\
\hline 2 & 0 & 4 & 0 & 0 & 0 & \\
\hline 3 & 1 & 3 & 5 & 0 & 0 & \\
\hline 4 & 0 & 0 & 0 & 1 & 0 & \\
\hline 5 & 0 & 0 & 1 & 1 & 3 & \\
\hline \multicolumn{6}{|c|}{ Orthodontist 3} & \multirow{6}{*}{$0.54(0.33-0.74)$} \\
\hline 1 & 3 & 1 & 0 & 0 & 0 & \\
\hline 2 & 1 & 3 & 0 & 0 & 0 & \\
\hline 3 & 1 & 3 & 0 & 4 & 1 & \\
\hline 4 & 0 & 0 & 2 & 1 & 0 & \\
\hline 5 & 0 & 0 & 0 & 1 & 2 & \\
\hline
\end{tabular}


methods referenced in the literature. A number of studies state that cervical vertebrae can be used as a reliable parameter for estimating skeletal age, but stress that this method should not be used exclusively and that further information should be acquired in order to reach a precise determination. ${ }^{4-7,12}$

In both the principal intraexaminer (radiologist) comparison and comparisons between the principal examiner and the orthodontists, the best results were obtained using the Baccetti et al. method. ${ }^{9}$ The authors propose a new classification, in which there are five maturation stages and variations in each stage, thereby allowing improved radiograph classification. As one maturation stage does not abruptly evolve into another, the Baccetti et al. method $^{9}$ eliminates many of the doubts as to which stage an individual belongs. In this method, denominated CVMS, only Stage III does not have a variation. The evaluators also agreed that the Baccetti et al. method $^{9}$ was easier to administer than the others, as the two anatomical variables in each stage simplify the classification. Although new, the validity of this method has been proven, and it has been used in a study aimed at developing a new formula for assessing the potential of mandibular growth. ${ }^{9}, 12-15$

On the other hand, the Seedat-Forsberg meth-

\section{References}

1. San Román P, Palma JC, Oteo MD, Nevado E. Skeletal maturation determined by cervical vertebrae development. Eur J Orthod. 2002 Jun;24(3):303-11.

2. Baccetti T, Franchi L, McNamara JA Jr. The cervical vertebral maturation (CVM) method for the assessment of optimal treatment timing in dentofacial orthopedics. Semin Orthod. 2005 Sep;11(3):119-29.

3. Generoso R, Sadoco EC, Armond MC, Gameiro GH. Evaluation of mandibular length in subjects with Class I and Class II skeletal patterns using the cervical vertebrae maturation. Braz Oral Res. 2010 Jan-Mar;24(1):46-51.

4. Flores-Mir C, Burgess CA, Champney M, Jensen RJ, Pitcher MR, Major PW. Correlation to skeletal maturation stages determined by cervical vertebrae and hand-wrist evaluations. Angle Orthod. 2006 Jan;76(1):1-5.

5. Gandini P, Mancini M, Andreani F. A comparison of handwrist bone and cervical vertebral analyses in measuring skeletal maturation. Angle Orthod. 2006 Nov;76(6):984-9. od $^{10}$ has a more simplified form, as it only assesses the C3 vertebra (its shape, the concavity on the lower edge, the $\mathrm{S}$-shape on the upper edge, the lipshape on the lower corners, and the rounding of the lower corners of the vertebral body) and uses the six stages proposed by Hassel and Farman. ${ }^{8}$ However, this method was the one that generated the greatest amount of doubt regarding the identification of the maturation stage. All evaluators stated that the method proposed by Seedat and Forsberg ${ }^{10}$ was the most difficult for identifying the skeletal maturation stage of orthodontic patients, reporting that the anatomical details viewed in this method are very subtle, thereby hindering their visualization. One alternative would be a combination of this method with another, such as that of Baccetti et al. ${ }^{9}$ Although this method achieved the least degree of agreement between evaluators, the results do not contraindicate its use.

\section{Conclusion}

The three methods demonstrated clinical applicability. However, the method proposed by Baccetti et al. ${ }^{9}$ achieved the best results, followed by the Hassel-Farman method ${ }^{8}$ and the Seedat-Forsberg method. $^{10}$

6. Soegiharto BM, Moles DR, Cunningham SJ. Discriminatory ability of the skeletal maturation index and the cervical vertebrae maturation index in detecting peak pubertal growth in Indonesian and white subjects with receiver operating characteristics analysis. Am J Orthod Dentofacial Orthop. 2008 Aug;134(2):227-37.

7. Lai EH, Liu JP, Chang JZ, Tsai SJ, Yao CC, Chen MH, et al. Radiographic assessment of skeletal maturation stages for orthodontic patients: hand-wrist bones or cervical vertebrae? J Formos Med Assoc. 2008 Apr;107(4):316-25.

8. Hassel B, Farman AG. Skeletal maturation evaluation using cervical vertebrae. Am J Orthod Dentofacial Orthop. 1995 Jan;107(1):58-66.

9. Baccetti T, Franchi L, McNamara JA Jr. An improved version of the cervical vertebral maturation (CVM) method for the assessment of mandibular growth. Angle Orthod. 2002 Aug;72(4):316-23. 
10. Seedat AK, Forsberg CD. An evaluation of the third cervical vertebra $(\mathrm{C} 3)$ as a growth indicator in Black subjects. SADJ. 2005 May;60(4):156,158-60.

11. Altman DG. Practical statistics for medical research. London: Chapman and Hall, 1991. 611 p.

12. Grave K, Townsend G. Cervical vertebral maturation as a predictor of the adolescent growth spurt. Aust Orthod J. 2003 Apr;19(1):25-32.

13. Santos EC, Bertoz FA, Arantes FM, Reis PM, Bertoz AP. Skeletal maturation analysis by morphological evaluation of the cervical vertebrae. J Clin Pediatr Dent. 2006 Spring;30(3):265-70.

14. Franchi L, Baccetti T, McNamara JA Jr. Mandibular growth as related to cervical vertebral maturation and body height. Am J Orthod Dentofacial Orthop. 2000 Sep;118(3):335340.

15. Caldas M de P, Ambrosano GM, Haiter Neto F. New formula to objectively evaluate skeletal maturation using lateral cephalometric radiographs. Braz Oral Res. 2007 OctDec;21(4):330-5. 